\title{
Comparison of in vivo toxicity, antioxidant and immunomodulatory activities of coconut, nipah and pineapple juice vinegars
}

\begin{abstract}
Background: Vinegar is widely used as a food additive, in food preparation and as a food supplement. This study compared the phenolic acid profiles and in vivo toxicities, and antioxidant and immunomodulatory effects of coconut, nipah and pineapple juice vinegars, which were respectively prepared via a two-step fermentation using Saccharomyces cerevisiae 7013 INRA and Acetobacter aceti vat Europeans. Results: Pineapple juice vinegar, which had the highest total phenolic acid content, also exhibited the greatest in vitro antioxidant capacity compared to coconut juice and nipah juice vinegars. Following acute and sub-chronic in vivo toxicity evaluation, no toxicity and mortality were evident and there were no significant differences in the serum biochemical profiles between mice administered the vinegars versus the control group. In the sub-chronic toxicity evaluation, the highest liver antioxidant levels were found in mice fed with pineapple juice vinegar, followed by coconut juice and nipah juice vinegars. However, compared to the pineapple juice and nipah juice vinegars, the mice fed with coconut juice vinegar, exhibited a higher population of $\mathrm{CD}^{+}$and $\mathrm{CD}^{+} \mathrm{T}$-lymphocytes in the spleen, which was associated with greater levels of serum interleukin- 2 and interferon- $\gamma$ cytokines. Conclusions: Overall, the data suggested that not all vinegar samples cause acute and sub-chronic toxicity in vivo. Moreover, the in vivo immunity and organ antioxidant levels were enhanced, to varying extents, by the phenolic acids present in the vinegars. The results obtained in this study provide appropriate guidelines for further in vivo bioactivity studies and pre-clinical assessments of vinegar consumption.
\end{abstract}

Keyword: Vinegar; Toxicity; Antioxidant; Immune response 